\title{
Evaluación de sensores de contenido de agua en el suelo para gestionar riegos de precisión
}

\author{
Vera, J., Conejero, W., Conesa, M.R., Mira-García, A.B., Ruiz-Sánchez, M.C. \\ Departamento de Riego, CEBAS-CSIC, Campus de Espinardo, 25, 30100 Murcia; jvera@cebas.csic.es; wenceslao@cebas.csic.es; mrcone- \\ sa@cebas.csic.es; abmira@cebas.csic.es; mcruiz@cebas.csic.es
}

Resumen: La escasez de agua y la necesidad de abastecer de alimentos de mayor calidad nutricional y organoléptica a una demanda cada vez más exigente y sensible por los sistemas de producción ha propiciado una gran tecnificación del riego. Esta tecnificación incluye el riego localizado, la fertigación y las mejoras en la toma de decisiones basadas en las necesidades hídricas del cultivo en cada etapa fenológica. Una de esas mejoras es la incorporación de sensores de contenido de agua para monitorizar la humedad del bulbo. En este trabajo se compara la respuesta de dos tipos de sensores comerciales de contenido volumétrico de agua en el suelo basados en la estimación de la constante dieléctrica: EnviroPro (capacitivo) y Acclima-TDR (Time Domain Reflectometry), instalados a 0,2, 0,4 y 0,6 $\mathrm{m}$ de profundidad y a 0,1 $\mathrm{m}$ del gotero, en una parcela de limeros (Citrus latifolia Tan. cv. Bearss) cultivados en meseta, en la finca experimental del CEBAS-CSIC (Santomera, Murcia) en un suelo franco-arcilloso. La gestión del riego localizado consistió en establecer umbrales de inicio y parada del riego basados en un agotamiento máximo permisible de agua en el perfil del suelo (MAD, de sus siglas en inglés Maximum Allowable Depletion) del 30\%. EL MAD considera una fracción de la reserva de agua útil, que varía desde capacidad de campo (CC) hasta el punto de marchitez (PM), parámetros estrechamente ligados a la textura del suelo. Los valores de contenido de agua en el suelo determinados con Acclima-TDR fueron ligeramente superiores a los obtenidos mediante el modelo matemático (dependiente de los porcentajes de arena, limo, arcilla y materia orgánica, y de la compactación del suelo): $0,35 \mathrm{~m}^{3} \mathrm{~m}^{-3}$ para capacidad de campo y $0,30 \mathrm{~m}^{3} \mathrm{~m}^{-3}$ para el $30 \%$ de MAD. Sin embargo, las lecturas correspondientes medidas con EnviroPro fueron de 0,54 y $0,38 \mathrm{~m}^{3} \mathrm{~m}^{-3}$ respectivamente. Además, las tendencias observadas en las curvas de agua en el suelo no fueron paralelas durante el ensayo, con una respuesta a la humectación y su redistribución más rápida en el caso de los sensores de capacitancia. De estos resultados cabe concluir que la gestión del riego de precisión requiere sensores cuyas características de exactitud, precisión y volumen de suelo explorado sean compatibles con el rango de MAD establecido (en este caso $0,04 \mathrm{~m}^{3} \mathrm{~m}^{-3}$ ), lo que se consiguió con los sensores TDR.

Palabras clave: Acclima-TDR; agua útil; EnviroPro; humedad del suelo; riego de precisión; MAD 


\title{
XXXVIII Congreso Nacional de Riegos CARTAGENA 2021
}

\section{Evaluation of soil water content sensors for precision irrigation management}

\author{
Vera, J., Conejero, W., Conesa, M.R., Mira-García, A.B., Ruiz-Sánchez, M.C. \\ Irrigation Departament, CEBAS-CSIC, Campus de Espinardo, 25, 30100 Murcia; jvera@cebas.csic.es; wenceslao@cebas.csic.es; mrcone- \\ sa@cebas.csic.es; abmira@cebas.csic.es; mcruiz@cebas.csic.es
}

\begin{abstract}
Water scarcity and the need to supply food of higher nutritional and organoleptic quality to an increasingly demanding and sensitive demand for production systems has led to a great technification of irrigation. This includes drip irrigation, fertigation and improvements in decision-making based on the crop water requirements at each phenological stage. One of these improvements is the use of water content sensors to monitor soil water status. This work compares the response of two types of commercial soil volumetric water content sensors based on dielectric constant estimation: EnviroPro (capacitive technique) and Acclima-TDR (Time Domain Reflectometry technique), installed at $0.2,0.4,0.6$ and $0.8 \mathrm{~m}$ depth and at $0.1 \mathrm{~m}$ from the dripper, in a plot of lime trees (Citrus latifolia Tan. $\mathrm{cv}$. Bearss) grown on ridges in the experimental field station of CEBAS-CSIC, in a clay loam soil. The management of drip irrigation consisted of establishing thresholds values, for start and stop irrigation, based on a Maximum Allowable Depletion (MAD) value of $30 \%$. MAD considers a fraction of the available water, which varies from field capacity (FC) to wilting point (WP), parameters that depend mainly on soil texture. The soil water content values determined with Acclima-TDR sensors showed light discrepancies with those derived from mathematical model (based on the percentages of sand, silt, clay and organic matter). In this case, the corresponding mean values were 0.35 and $0.30 \mathrm{~m}^{3} \mathrm{~m}^{-3}$ for FC and 30\% MAD, respectively, while they were 0.54 and $0.38 \mathrm{~m}^{3} \mathrm{~m}^{-3}$ for EnviroPro probes. In addition, the trends observed in the soil water content curves were not parallel during the studied period, with a faster response to wetting and water re-distribution in the capacitance sensors. From these results it can be concluded that precise irrigation management requires sensors whose accuracy, precision and soil volume exploration characteristics was compatible with the established MAD range (0.04 $\mathrm{m}^{3} \mathrm{~m}^{-3}$ in our conditions). This was accomplished with the TDR sensors.
\end{abstract}

Keywords: Acclima-TDR; available soil water; EnviroPro; precision irrigation; soil water content; MAD 


\section{Congreso Nacional de Riegos CARTAGENA 2021}

\section{Introducción}

La limitación de disponibilidad de recursos hídricos para el riego ha propiciado la tecnificación de riego localizado y el fertiriego, lo que junto con la información derivada de la monitorización del estado hídrico del suelo y de la planta ha acuñado el concepto de Riego de Precisión.

Paralelamente, el estado hídrico del suelo ha servido de referencia para manejar el riego, tanto por el estado energético, mediante tensiómetros, como por el contenido de agua, estimado con sensores [1]. Este último ha experimentado un gran auge en los últimos 30 años gracias al desarrollo de la microelectrónica de sensores inalámbricos y la transmisión de datos al usuario para la toma de decisiones [2].

La tecnología más extendida de monitorización del contenido de agua en el suelo $\left(\theta_{\mathrm{v}}\right)$ se basa en la estimación de la humedad volumétrica a partir de la constante dieléctrica, empleando técnicas capacitivas, como es el caso de los sensores EnviroScan, EnviroPro, Aquacheck, C-probe, Decagon, Teros, o Arifruit. Estos sensores permiten observar el perfil de humedad a diferentes profundidades y derivar el stock hídrico de agua en el suelo. Típicamente la traza de $\theta_{\mathrm{v}}$ entre dos riegos muestra un patrón de escalera descendente en el que durante el día se produce el descenso y por la noche se estabiliza. Esta tendencia ha servido para establecer valores umbrales de inicio y parada del riego de forma gráfica.

Sin embargo, para la determinación cuantitativa del stock hídrico es necesario un laborioso trabajo de calibración que depende del tipo de suelo, su densidad aparente, contenido de materia orgánica y propiedades estructurales.

Otra tecnología para estimar el contenido de agua en el suelo es la reflectometría en el dominio del tiempo (de sus siglas en inglés TDR, time domain reflectometry), desarrollada por Topp [3] y que estima con mayor precisión el tiempo de propagación de un pulso eléctrico en el suelo y permite obtener la constante dieléctrica virtualmente independiente de la textura, salinidad y demás propiedades del suelo. Sin embargo, el coste de estos equipos y su consumo energético no han conseguido un uso extensivo en la agricultura de regadío. Recientemente, esta tecnología se ha miniaturizado mediante nano-electrónica (sensores Acclima), alcanzando una precisión igual o mayor a la de sus predecesores con un coste similar al de los sensores capacitivos.

En este trabajo se presentan los resultados de un ensayo de riego en limeros cv. Bearss cultivados en meseta en una parcela de la finca experimental del CEBAS-CSIC en Santomera (Murcia), monitorizada con sensores capacitivos (EnviroPro) y sensores TDR (Acclima-TDR).

\section{Materiales y métodos}

El ensayo se llevó a cabo durante los meses de verano de 2021 en la finca experimental del CEBAS-CSIC en Santomera (Murcia), en una parcela de 1,2 ha de limeros (Citrus latifolia Tan., cv. Bearss) de 5 años de edad, injertados sobre Citrus macrophylla L., cultivados en meseta, con un marco de plantación de $6 \mathrm{~m} \times 5 \mathrm{~m}$. El suelo es pedregoso, altamente calcáreo (45\% de carbonato cálcico), de textura franco-arcillosa: Arena $40 \%$ p/p, Limo $28 \%$ p/p, Arcilla 32\% p/p con una composicion de 17\% esmectita, $29 \%$ polirgoskita, $41 \%$ illita-mica, $8 \%$ caolinita y $5 \%$ clorita (arcillas tipo 2:1, lo que favorece el desarrollo de grietas en el suelo) y el contenido de materia orgánica fue bajo (1,4\%). El sistema de riego localizado consistió en una doble línea porta-goteros separada $1 \mathrm{~m}$, con 4 goteros autocompensantes de $4 \mathrm{~L} \mathrm{~h}^{-1}$ por árbol, situados equidistantes a 0,75 m del tronco.

La parcela se equipó con los siguientes sensores de contenido de agua en el suelo: EnviroPro, sonda encapsulada capacitiva de 0,8 m tipo "drill and drop" (Entelechy Pty Ltd, Australia) y 


\section{Congreso Nacional de Riegos CARTAGENA 2021}

Acclima-TDR modelo 315H (Acclima, Inc., USA). Ambos sensores se instalaron a 0,1 m del gotero más cercano al tronco y a las profundidades de 0,2, 0,4, 0,6, 0,8 $\mathrm{m}$ (Figura 1) en 4 árboles representativos de la parcela experimental.

A

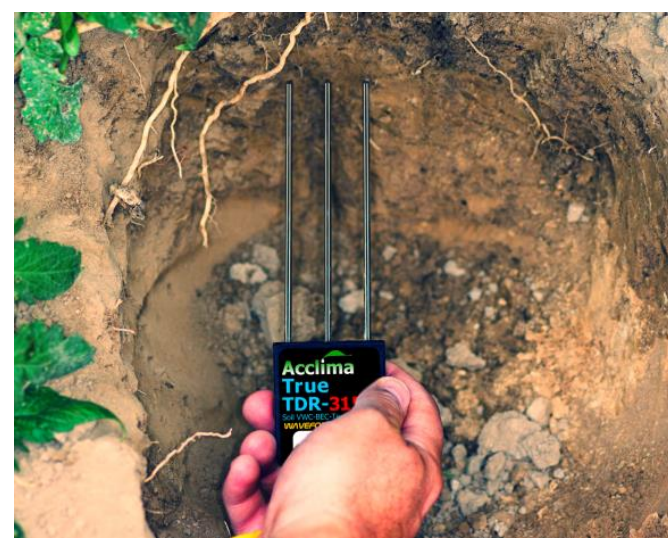

B

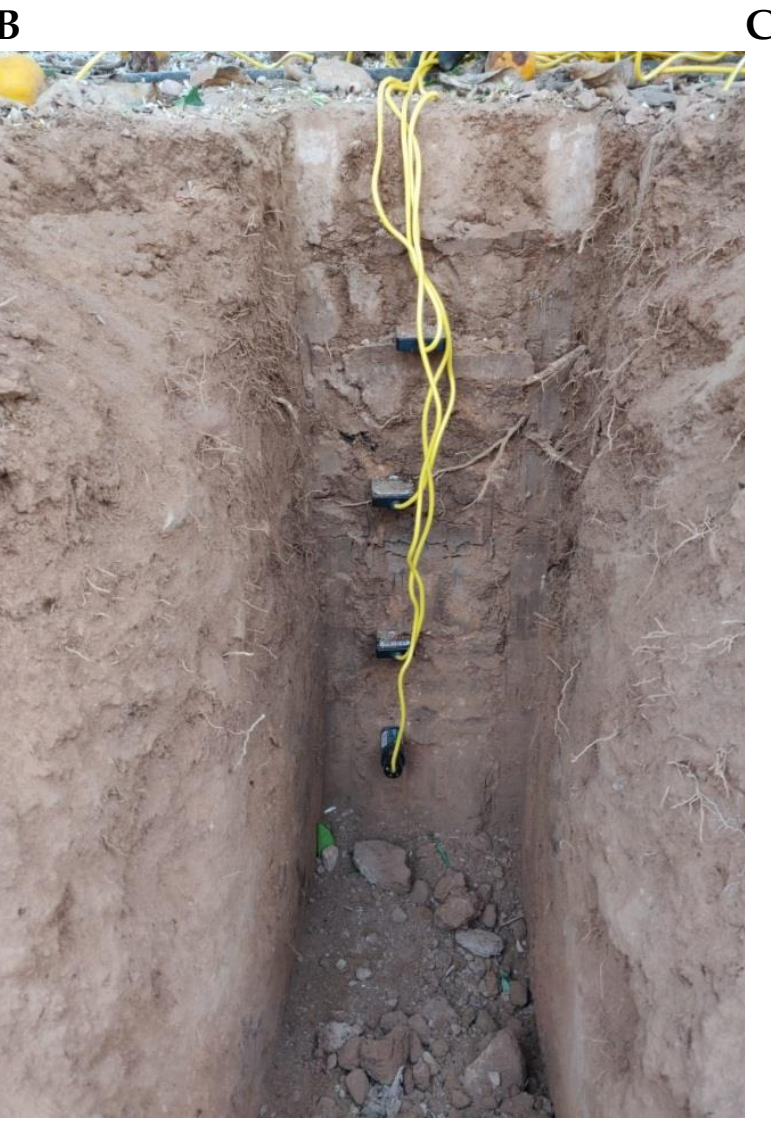

C

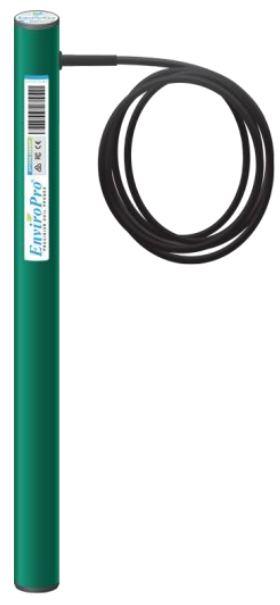

Figura 1. (A) Sensor Acclima-TDR utilizado; (B): Disposición vertical de los sensores Acclima-TDR en el perfil de suelo seleccionado; (C): Sonda EnviroPro de 0,8 m de longitud utilizada.

La programación del riego se realizó de forma automática mediante una red inalambrica de sensores y actuadores (WSAN, Wireless Sensor and Actuator Network). Los sensores permitieron monitorizar en continuo $\theta_{\mathrm{v}}$ en el perfil $0-0,8 \mathrm{~m}$ del suelo y actuar en la apertura/cierre de las electroválvulas. Para ello se establecieron valores umbrales de $\theta_{\mathrm{v}}$ correspondientes a un $30 \%$ de MAD y capacidad de campo para el inicio y fin del riego, respectivamente. El MAD (de sus siglas en inglés Maximum Allowable Depletion) considera una fracción de la reserva de agua útil del suelo, definida como CC menos PM; ambos parámetros estrechamente ligados a la textura del suelo. El sistema de telemetría tomaba lecturas cada $5 \mathrm{~min}$. y registraba el promedio cada $15 \mathrm{~min}$. Las unidades de radio-transmisión enviaban datos al servidor web addVANTAGE (ADCON Telemetry, Austria) para el procesamiento y la visualización de los datos.

De forma previa a la toma de datos, en el caso de la sonda EnviroPro se realizó un ensayo de calibración en laboratorio. Para ello se tomó una muestra de suelo de la parcela que fue tamizado ( $2 \mathrm{~mm})$ y secado en estufa a $105{ }^{\circ} \mathrm{C}$ durante $48 \mathrm{~h}$. El suelo seco se introdujo en cilindros de PVC de $150 \mathrm{~mm}$ de diámetro interno y $190 \mathrm{~mm}$ de altura, compactándose a 1,3-1,6 t m³, haciendo uso para ello de una 


\section{Congreso Nacional de Riegos CARTAGENA 2021}

prensa hidráulica de puente (MECAMAQ). Periódicamente se fueron añadiendo 100 cc de agua de riego hasta producir el drenaje de la columna de suelo.

En el caso del sensor Acclima-TDR, que no precisa de calibración, mide directamente $\theta_{\mathrm{v}}$ a partir de la constante dieléctrica y de la aplicación de la ecuación de Topp [3], ya que no está afectado por la composición mineral ni la estructura del suelo.

\section{Resultados y discusión}

La aplicación del modelo matemático de Saxton y Rawls [4] en las condiciones texturales de diseño obtuvo valores de $0,46 \mathrm{~m}^{3} \mathrm{~m}^{-3}$ (saturación), 0,33 $\mathrm{m}^{3} \mathrm{~m}^{-3}$ (CC) y $0,20 \mathrm{~m}^{3} \mathrm{~m}^{-3}(\mathrm{PM})$, siendo los valores de referencia para la comparación de los distintos sensores estudiados (Figuras 2, 3 y 4).

La curva de calibración realizada en laboratorio de la sonda EnviroPro mostró una relación lineal $y=0,83 x+14,37\left(r^{2}=0,91^{* *}\right)$, donde $y$ es el valor de $\theta_{\mathrm{v}}$ del sensor y $x$ el valor de $\theta_{\mathrm{v}}$ real correspondiente. La densidad aparente del suelo fue determinante en los valores observados por la sonda EnviroPro (Figura 2). Hasta un valor de $\theta_{\mathrm{v}}$ de $0,2 \mathrm{~m}^{3} \mathrm{~m}^{-3}$, las pendientes fueron muy diferentes entre sí, sin un orden lógico por el grado de compactación, posiblemente debido a la presencia de arcillas expandibles que facilitaron la aparición de grietas impidiendo una buena uniformidad de agua en el perfil del cilindro de suelo empleado en la calibración. A partir de valores de humedad superiores, la respuesta de la sonda varió hasta un 50\% según la densidad aparente del suelo, si bien la pendiente en todas las curvas de retención de humedad fue prácticamente la misma, limitadas tan solo en el nivel máximo de agua admisible, mayor cuanto menor fue la densidad aparente.

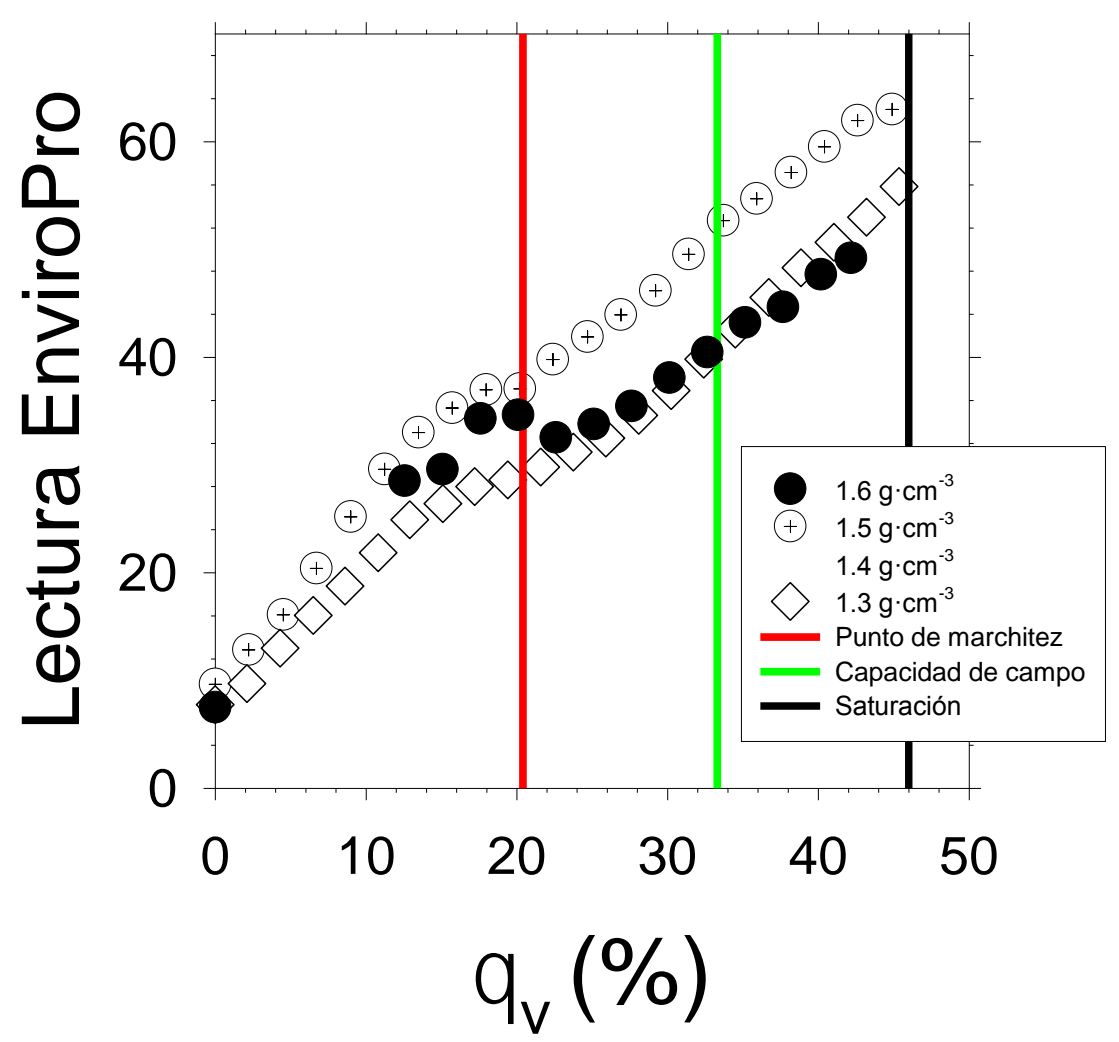

Figura 2. Calibración en laboratorio de sensores EnviroPro, utilizando columnas de PVC (150 mm diámetro y 190 mm alto) rellenas de suelo franco-arcilloso de diferente densidad aparente. 


\section{Congreso Nacional de Riegos CARTAGENA 2021

Las medidas de $\theta_{\mathrm{v}}$ realizadas en campo con sondas EnviroPro, previamente calibradas, generaron un valor de CC de $0,54 \mathrm{~m}^{3} \mathrm{~m}^{-3}$, discrepando de los valores observados en laboratorio (Figura 3). Igualmente, se constató la debilidad en la determinación cuantitativa de $\theta_{\mathrm{v}}$ para el valor de MAD = $30 \%$, que correspondía al umbral establecido como criterio de control para el inicio del riego (Figura 3). Este hecho evidencia los errores derivados de la interpretación de valores absolutos cuando se utiliza esta sonda.

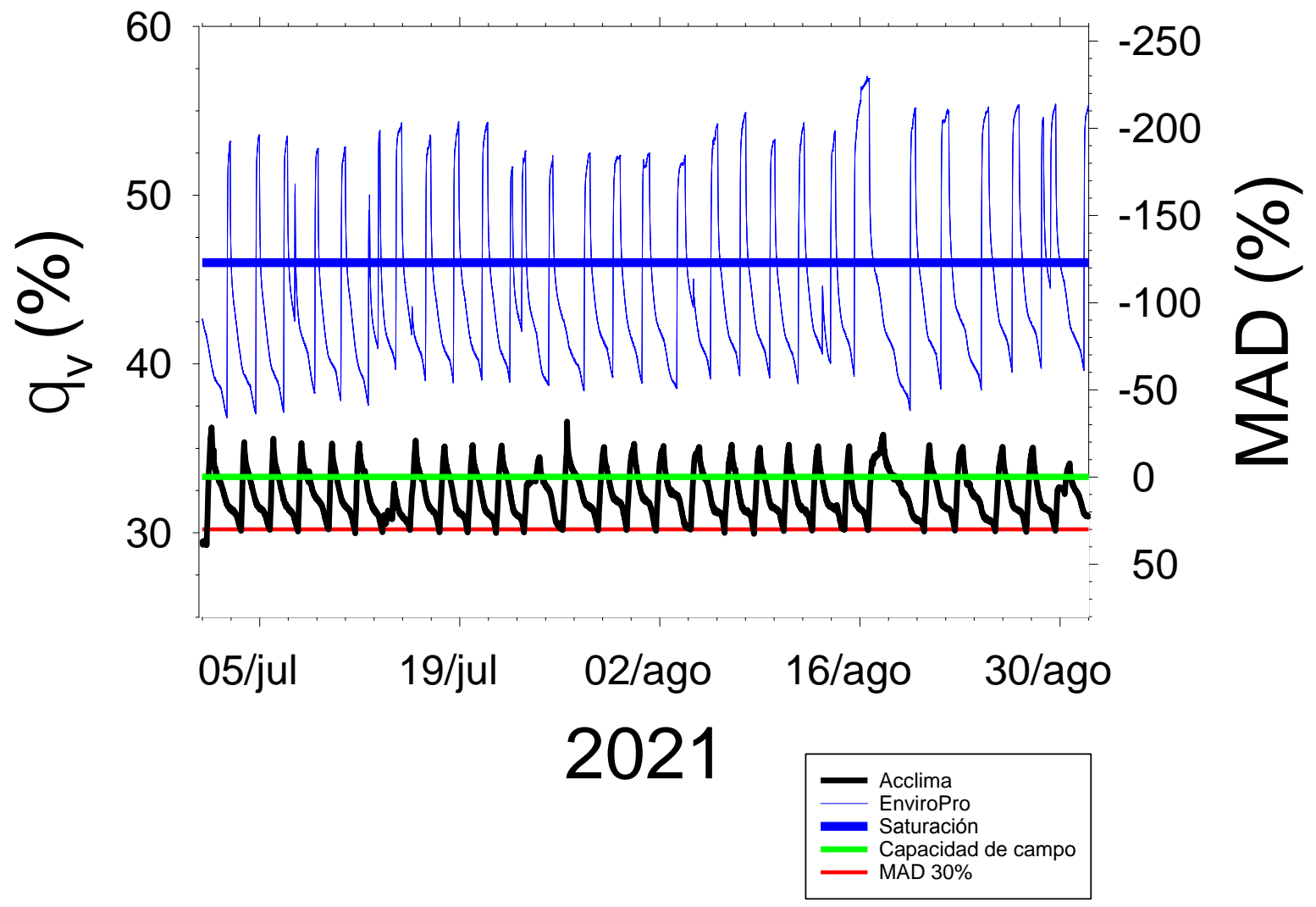

Figura 3. Evolución del contenido medio de agua en el suelo $\left(\theta_{\mathrm{v}}\right)$ monitorizado con sensores EnviroPro y Acclima-TDR en el perfil 0-0,6 m de suelo de la parcela experimental. Las líneas horizontales indican los valores umbrales de $\theta_{\mathrm{v}}: 30 \% \mathrm{MAD}$ y capacidad de campo, para iniciar y detener el riego, respectivamente, y el valor de saturación.

Adicionalmente, el concepto de "tendencia" de la curva de $\theta_{\mathrm{v}}$ fue durante muchos años el criterio empleado para iniciar el riego, ya que éste muestra de forma clara las extracciones de agua por la planta y/o el drenaje durante el periodo diurno y su estabilización durante la noche (Figura 4). 


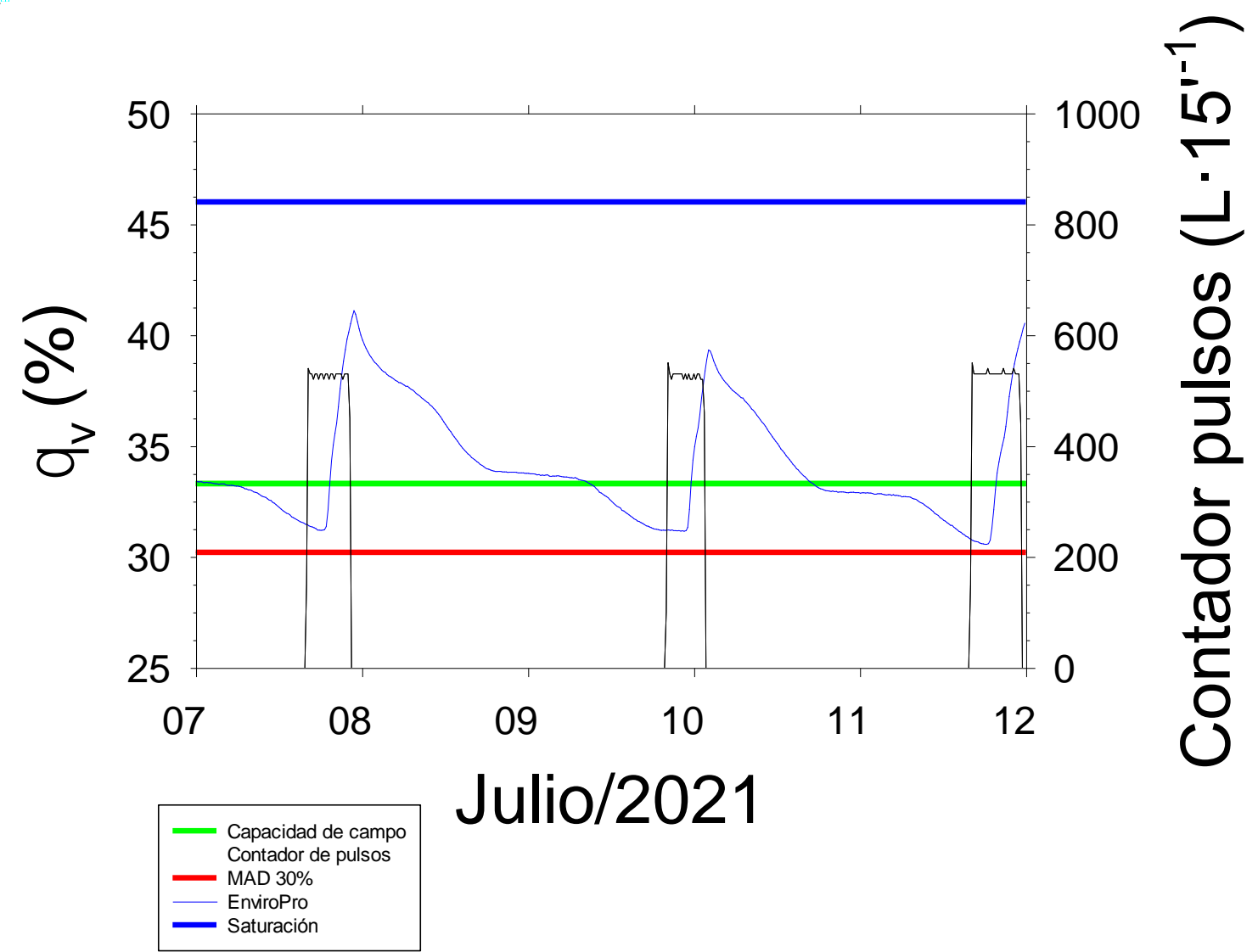

Figura 4. Evolución del contenido volumétrico medio de agua $\left(\theta_{\mathrm{v}}\right)$ en el perfil 0-0,6 m, medido con sonda EnviroPro y los eventos de riego (barras). Las líneas horizontales indican los valores 30\% MAD, capacidad de campo y saturación.

Las medidas de $\theta_{\mathrm{v}}$, realizadas con sensores Acclima-TDR en campo, mostraron valores de CC de $0,35 \mathrm{~m}^{3} \mathrm{~m}^{-3}$, demostrando una ligera diferencia con los valores del modelo de Saxton y Rawls (Figura 3). Este valor de CC fue utilizado como umbral para finalizar el riego en las condiciones experimentales.

Las discrepancias entre las medidas de saturación y capacidad de campo, realizadas en laboratorio y en condiciones de campo, han sido demostradas por Evett et al. [5], quienes propusieron el valor de $\theta_{\mathrm{v}}$ máximo observado en campo como referencia a partir de la cual establecer la dinámica de extracción de agua por la planta durante el ciclo de cultivo.

La exactitud de la medida del contenido de agua en el suelo para satisfacer un MAD del 30\% supuso, en las condiciones experimentales, una franja muy estrecha de $\theta_{\mathrm{v}}\left(0.04 \mathrm{~m}^{3} \mathrm{~m}^{-3}\right)$, lo que condiciona la viabilidad del riego de precisión.

No obstante, es importante señalar que la adopción del concepto de MAD para establecer umbrales de inicio del riego permite la generalización de los niveles de agotamiento de agua en el suelo independientemente de sus características, y su viabilidad ha sido constatada por medio de la determinación de valores de potencial hídrico de tallo, indicadores de un adecuado suministro hídrico en las plantas de limero estudiadas [6]. 


\section{Congreso Nacional de Riegos CARTAGENA 2021}

\section{Conclusiones}

- La calibración de las sondas capacitivas EnviroPro requiere un trabajo laborioso especifico de campo para cada una de las condiciones texturales de suelo. Ello se demostró con las importantes discrepancias observadas en los valores de $\theta_{\mathrm{v}}$ derivados de la calibración realizada en laboratorio. En el caso de los sensores Acclima-TDR, el trabajo previo de laboratorio no es necesario y la lectura realizada en campo mostró valores de $\theta_{\mathrm{v}}$ exactos, salvo en algún caso, por defectos en la instalación del sensor ocasionados por la presencia de piedras.

- El valor de CC obtenido con sondas EnviroPro resultó más diferente del derivado del modelo de Saxton y Rawls que el obtenido con sensores Acclima-TDR.

- El concepto de MAD para el riego de precisión permite la generalización de los niveles de agotamiento de agua en el suelo independientemente de sus características, así como su validación mediante el potencial hídrico de tallo.

- El ensayo de campo realizado permite concluir que ambos sensores (capacitivos y TDR) permiten observar la tendencia de $\theta_{\mathrm{v}}$ en el perfil del suelo. Sin embargo, el riego de precisión requiere de valores reales, exactos y precisos de $\theta_{\mathrm{v}}$ para su aplicación.

- El valor de MAD establecido supuso un valor de $\theta_{\mathrm{v}}=0,04 \mathrm{~m}^{3} \mathrm{~m}^{-3}$ inferior a $C C$, lo que resulta inconsistente cuando se utilizan sensores con un error superior a este valor.

- La nueva generación de sensores TDR encapsulados supone una mayor exactitud y precisión en la medida de $\theta_{\mathrm{v}}$, con respecto a sus antecesores. Además, exploran un volumen de suelo mayor y tienen un precio similar al de los sensores capacitivos.

\section{Agradecimientos}

El trabajo se ha financiado con el proyecto PID2019-106226RB-C2-1 (Plan Nacional AEI) y 19903/GERM/15 (Fundación Séneca, Región de Murcia). M.R. Conesa agradece la ayuda posdoctoral disfrutada del programa Juan de la Cierva (FJCI-2017-32045).

\section{Referencias}

1. Merriam, J.L. A management control concept for determining the economical depth and frequency of irrigation. Transactions of ASAE 1966, 9: 492-498. doi:10.13031/2013.40014

2. Vera J., Conejero, W., Mira-Garcia, A.B., Conesa, M.R., Ruiz-Sánchez, M.C. Towards irrigation automation based on dielectric soil sensors. J. Hort. Sci. and Biotech. 2021, https://doi.org/10.1080/14620316.2021.1906761

3. Topp, G.C., Davis, J.L., Annan, A.P. Electromagnetic determination of soil water content: Measurements in coaxial transmission lines. Water Resources Research, 1980, 16, 574-582. doi:10.1029/WR016i003p00574T

4. Saxton, K.E., Rawls, W. Soil water characteristics. Hydraulic properties calculator. 2009 https://hrsl.ba.ars.usda.gov/soilwater/Index.htm

5. Evett, S.R., Stone, K.C., Schwartz, R.C., O'Shaughnessy, S.A., Colaizzi, P.D., Anderson, S.K., Anderson, D.J. Resolving discrepancies between laboratory-determined field capacity values and field water content observations: implications for irrigation management. Irrig. Sci. 2019, 37(6)

6. Mira-Garcia, A.B., Conejero, W., Vera, J., Ruiz-Sánchez, M.C. Leaf water relations in lime trees grown under shade netting and open-air. Plants. 2020, 9, 510; doi:10.3390/plants9040510 